\title{
The SORCE SIM Solar Spectrum: Comparison with Recent Observations
}

\author{
J.W. Harder · G. Thuillier · E.C. Richard • S.W. Brown • \\ K.R. Lykke • M. Snow • W.E. McClintock • \\ J.M. Fontenla $\cdot$ T.N. Woods $\cdot$ P. Pilewskie
}

Received: 28 October 2009 / Accepted: 4 April 2010 / Published online: 20 April 2010

(C) The Author(s) 2010. This article is published with open access at Springerlink.com

\begin{abstract}
The Spectral Irradiance Monitor (SIM) on board the NASA SORCE satellite (Solar Radiation and Climate Experiment) was launched on 25 January 2003 and has been making twice-daily measurements of solar variability in the 220 to $1630 \mathrm{~nm}$ range and daily measurements in the 1600 to $2400 \mathrm{~nm}$ range. This study presents preflight and postlaunch calibration activities of the SIM instrument and its flight spare components as well as inflight comparisons with the ATLAS 3 composite spectrum (Atmospheric Laboratory for Applications and Science) in the ultraviolet (UV), visible, and near infrared (NIR) as well as comparisons with the SOLSTICE (Solar Stellar Irradiance Comparison Experiment) in the UV. In the 258 to $1350 \mathrm{~nm}$ range, the SIM agrees with ATLAS 3 with a fractional difference of $-0.021 \pm 0.021$ ( $k=1$, estimated standard deviation) and with the additional corrections discussed herein the agreement improves to $-0.008 \pm 0.021(k=1)$. In the ultraviolet (220-307 nm) the agreement between all the instruments in this study is better than $5 \%$, but fractional differences reveal other instrument- and calibration-related differences. In the 1350 to $2400 \mathrm{~nm}$ range the agreement between SIM and ATLAS 3 is about 8\%, so these SIM data are corrected to agree with ATLAS 3 in this range.
\end{abstract}

\section{Introduction}

With the continuing interest of the Sun's role in understanding the sources of Earth climate variability (Rind et al., 2008), accurate and precise measurements of the solar spectrum

J.W. Harder (凶) · E.C. Richard · M. Snow - W.E. McClintock · J.M. Fontenla - T.N. Woods ·

P. Pilewskie

Laboratory for Atmospheric and Space Physics (LASP), University of Colorado, Boulder, CO, USA

e-mail: jerald.harder@lasp.colorado.edu

G. Thuillier

LATMOS du CNRS, Bp 3, 91371 Verrieres le Buisson, France

S.W. Brown · K.R. Lykke

Optical Technology Division, National Institute of Standards and Technology (NIST),

Gaithersburg, MD, USA 
remain an essential component of this research effort. Knowledge of the solar irradiance variability allows us to investigate the responsivity of climate models to such changes. Furthermore, the absolute irradiance is necessary to calculate, for example, the concentration of certain atmospheric species, and compare them with observations to evaluate the accuracy of the atmospheric model predictions. Currently, only the SIM and the ENVISAT SCIAMACHY (Pagaran, Weber, and Burrows, 2009) are making routine daily measurements of solar variability, and the SOLAR instrument onboard the International Space Station (Thuillier et al., 2009) is continuing the SOLSPEC data heritage. The SOLAR instrument observes the Sun continuously about two weeks per month, and internal instrument calibrations and performance checks are performed when a view of the Sun is not possible. Detailed comparisons of the time series of these instruments will be addressed in future publications.

Through a series of well-documented calibration papers, Thuillier and co-workers (Thuillier et al. 1997, 1998a, 1998b, 2003; Mandel et al., 1998) have presented the calibration and flight observations of the SOLar SPECtrum (SOLSPEC) instrument for its Spacelab (1982), ATmospheric Laboratory for Applications and Science (ATLAS) missions 1-2 - 3 flights (1992 - 1994), and the SOlar SPectrum (SOSP) instrument on the EURECA mission (1993 - 1994). This effort has established the SOLSPEC spectrum as the de facto standard for solar spectral irradiance in the 400 to $2400 \mathrm{~nm}$ range. Figure 1a shows the SIM and the ATLAS 3 composite spectra with Figure $1 \mathrm{~b}$ giving the quoted resolution of the instruments. Figure 1c shows the ranges of the SIM detectors and the instruments used to construct the composite. The details on this construction are discussed in Thuillier et al. (2004). The comparisons in this paper are performed using the ATLAS 3 spectrum as the standard reference since it establishes the reference solar minimum state of the Sun for solar cycle 22. The differences between ATLAS 1 and 3 are confined to wavelengths less than $410 \mathrm{~nm}$ and the visible and infrared portions of the two composites are identical. Differences for the ultraviolet portion of the spectrum for the two composites are mostly due to solar variability between the high solar activity ATLAS1 time period (March 1992) and the quiet Sun ATLAS3 period (November 1994).

Recently, Woods et al. (2009) published the findings of the Solar Irradiance Reference Spectrum (SIRS), a solar cycle 23 analog to the ATLAS 3 composite acquired during a quiescent solar minimum period in April of 2008. The SIRS spectrum, which covers the 2 to $2400 \mathrm{~nm}$ range with very few spectral gaps, was constructed from near simultaneous observations by the TIMED SEE XUV (Thermosphere-Ionosphere-Mesosphere-EnergeticsDynamics Extreme Ultraviolet spectrometer), SORCE SOLSTICE and SIM instruments (Solar Radiation and Climate Experiment, SOLar STellar Irradiance Comparison Experiment and Spectral Irradiance Monitor), and the first results from SDO EVE (Solar Dynamics Observatory, EUV Variability Experiment) rocket calibration instrument (Chamberlin et al., 2009). Comparisons from SIRS and ATLAS 3 are briefly presented in Woods et al. (2009).

The SIM instrument was developed to replace and extend the operating range of the Upper Atmospheric Research Satellite (UARS) SOLSTICE $N$-channel spectrometer (Rottman, Woods, and Sparn, 1993) that was used to study solar variability in the 280 to $420 \mathrm{~nm}$ region. Analysis of the UARS $N$-channel spectrometer measurements showed a long-term precision of about 0.1 to $1 \%$ because the calibration stars were too dim in this spectral region to permit an adequate degradation correction. This compromised its ability to determine the solar cycle variations in the near UV that were estimated during the UARS era (solar cycle 22) to be on the order of $0.5-0.1 \%$ in the 300-420 nm range (Lean et al., 1997). In the visible for wavelengths longer than $400 \mathrm{~nm}$, solar cycle changes are even smaller, on the order of $0.1 \%$, as inferred from space-based observations during solar cycle 23 (Pagaran, Weber, and Burrows, 2009; Harder et al., 2009). Because of this need to understand the nature of 


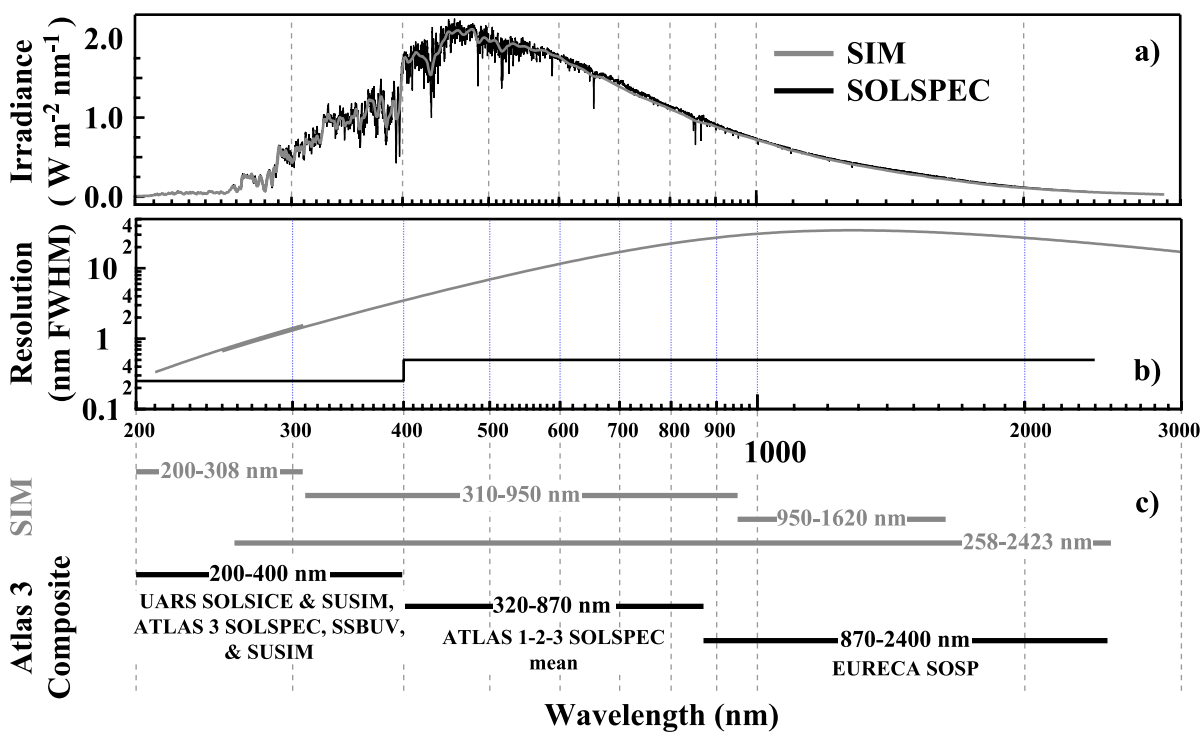

Figure 1 The ATLAS 3 and SIM spectra. Panel (a) shows the full measurement range of the SIM instrument and the composite; SIM is shown in gray and SOLSPEC in black in all three panels. Panel (b) shows the resolution of the SIM and SOLSPEC instruments, and panel (c) shows the detector ranges for SIM and the individual instruments and the wavelength ranges used to construct the composite.

solar variability throughout the visible/infrared spectral range, the SORCE SIM instrument was developed to provide very broad spectral coverage, high measurement precision, and the ability to perform physically based degradation corrections to about the 0.1 to $0.01 \%$ level (depending on wavelength).

This paper has the following structure. Section 2 discusses the SIM instrument optical design, its characterization, and data products. Of particular interest are Sections 2.2.1 that discusses the pre-launch component level calibration of the instrument and its validation with an FEL lamp, and Section 2.2.2 describes the postlaunch laboratory studies of critical flight spare components such as the ESR (Electrical Substitution Radiometer) detector and the prism instrument line shape. These postlaunch experiments address shortcomings in the preflight calibration and were conducted at the NIST SIRCUS facility (Spectral Irradiance and Radiance Calibration with Uniform Sources, Brown et al., 2004). Section 3 describes the instrument, calibration, and data of the SOLSPEC instrument and establishes the usage of the ATLAS 3 composite spectrum as the reference for the comparative study of in-flight instruments in Section 4. Section 4.1 presents a detailed comparison of the in-flight measurements of SIM with the ATLAS 3 reference spectrum. This is done in the $258-1350 \mathrm{~nm}$ region without applying the corrections found is Section 2.2. Similarly, Section 4.2 discusses the comparisons in the 220 to $307 \mathrm{~nm}$ region that includes the UARS and SORCE SOLSTICE instruments. In Section 4.3 the correction factor derived in Section 2.2 is applied for SIM data in the 258 to $1350 \mathrm{~nm}$ range and the results of this correction are evaluated. In this way, these additional calibration activities provide an independent assessment of SIM calibration in the 258 to $1350 \mathrm{~nm}$ range and the application of the corrections found from these studies brings the two instruments into agreement at the $2 \%$ level. This section also shows that for wavelengths greater than $1000 \mathrm{~nm}$ the agreement between the SIM and ATLAS 3 diverge with the SIM results becoming systematically lower with increasing wavelength 
out to $1500 \mathrm{~nm}$ where the disagreement is about $8 \%$ and remains so out to $2400 \mathrm{~nm}$. The SIM calibration is less certain than that of SOLSPEC for $\lambda>1000$; therefore, we adopt the SOLSPEC values for the longer wavelength region.

\section{SIM Spectrometer and Data}

\subsection{Optical Design}

The SIM instrument is based on the Féry prism design that allows for focus and dispersion with only one refractive optical element (Warren, Hackwell, and Gutierrez, 1997). This attribute is critical for degradation corrections over the course of the now 6.5-year mission. The spectrometer design has the following attributes: $i$ ) it produces a relatively flat focal plane, so multiple detectors can be used with equally good imaging quality, $i$ ) it achieves broad wavelength coverage without order sorting optics, and iii) it has a very high scattered light rejection ratio $\left(>10^{4}\right)$. The major disadvantage of the design is a non-linear dispersion function as depicted in Figure 1b. Thus instrument comparisons like the ones described herein require detailed slit function convolution to permit instrument comparison (see Section 4). SIM employs four detectors in its focal plane (Figure 1c): $n-p$ silicon photodiodes are used to record the UV and visible wavelengths (200-308 and 310-950 nm, respectively), an InGaAs photodiode covers the 950 to $1620 \mathrm{~nm}$ range, and an ESR covers the 258 to $2423 \mathrm{~nm}$ range. The ESR is used to re-calibrate the radiometric sensitivity of the photodiodes over their operating ranges and to provide the 1620 to $2423 \mathrm{~nm}$ daily data product. Since the SIM instrument is a scanning spectrometer, the instrument profile (point spread function) is nominally triangular, but small changes in magnification actually cause it to have a trapezoidal shape; a small amount of coma and near-in edge scattering induce additional structure to the profile at the $0.5 \%$ level. For data processing, ray trace analysis is used to generate the instrument profile at all wavelength positions and these modeled instrument profile data are then verified against tunable laser scans (see Section 2.2.2 for further discussion).

\subsection{Characterization}

\subsubsection{Pre-Flight Calibration}

The calibration approach adopted for the SIM investigation uses calibrations of the instrument components (e.g. detector sensitivity, prism transmission, and entrance slit area) as inputs to a measurement equation that determines the end-to-end instrument calibration. This process was validated by performing an independent end-to-end calibration using an irradiance standard lamp (referred to as an FEL lamp), which was calibrated at the National Institute of Standards and Technology (NIST). Since the SIM instrument is designed to measure and monitor solar variability, the detector gains were set to accommodate solar intensities. An FEL lamp set to illuminate the SIM with $a \approx 0.5^{\circ}$ beam to simulate the solar input produces only a few percent of the irradiance of the Sun; thus these sources can only verify the unit level calibration and identify issues related to the preflight component level calibration.

In its most compact form, measured spectral irradiance $E_{\lambda}$ at the set wavelength $\left(\lambda_{s}\right)$ can be expressed as measurement equations for the ESR and photodiode detector currents 
Table 1 Uncertainty contributions to the determination of the SIM absolute accuracy.

\begin{tabular}{|c|c|c|c|c|}
\hline Term (unit) & Symbol & Value/range & Uncertainty $(k=1)$ & Derived from \\
\hline Wavelength (nm) & $\lambda$ & $201-2423$ & $0.2 \pm \lambda \times\left(150 \times 10^{-6}\right)$ & $\begin{array}{l}\lambda \text { standards, solar } \\
\text { spectrum }\end{array}$ \\
\hline ESR power $(\mathrm{W})$ & $E_{\mathrm{ESR}}$ & $1 \times 10^{-7}-5 \times 10^{-5}$ & $\approx 2 \times 10^{-9} \mathrm{nWHz}^{-1 / 2}$ & Detector testing \\
\hline $\begin{array}{l}\text { Entrance slit area } \\
\left(\mathrm{mm}^{2}\right)\end{array}$ & $A_{\text {slit }}$ & 2.1 & $5 \times 10^{-5}$ & Slit diffraction \\
\hline \multirow[t]{2}{*}{$\begin{array}{l}\text { ESR optical } \\
\text { efficiency }(\%)\end{array}$} & \multirow[t]{2}{*}{$\alpha_{\lambda}$} & \multirow[t]{2}{*}{$100 \%$} & $\begin{array}{l}+0 \text { to }-2 \% \\
(200-1000 \mathrm{~nm})\end{array}$ & \multirow[t]{2}{*}{$\begin{array}{l}\text { SIRCUS, flight } \\
\text { spare ESR }\end{array}$} \\
\hline & & & $\begin{array}{l}+0 \text { to }-10 \% \\
(1000-2700 \mathrm{~nm})\end{array}$ & \\
\hline $\begin{array}{l}\text { Photodiode } \\
\text { radiant sensitivity } \\
\left(\mathrm{AW}^{-1}\right)\end{array}$ & $R_{\lambda}$ & $0.08-1.0$ & $\begin{array}{l}2-4 \% \\
\text { (wavelength dependent) }\end{array}$ & $\begin{array}{l}\text { In-flight } \\
\text { comparisons with } \\
\text { ESR }\end{array}$ \\
\hline $\begin{array}{l}\text { Prism } \\
\text { transmission }(\%)\end{array}$ & $T_{\lambda}$ & $0.55-0.77$ & $\begin{array}{l} \pm 0.1 \% 200-700 \mathrm{~nm} \\
\geq \pm 1 \% 700-2700 \mathrm{~nm}\end{array}$ & $\begin{array}{l}\text { Laboratory } \\
\text { measurements } \\
\text { (see Harder } \text { et al. } \\
\text { 2005a) }\end{array}$ \\
\hline $\begin{array}{l}\text { Diffraction loss } \\
(\%)\end{array}$ & $\Phi_{\lambda}$ & $0.3-2.2$ & $\approx 0.01$ & $\begin{array}{l}\text { Diffraction } \\
\text { theory }\end{array}$ \\
\hline $\begin{array}{l}\text { Instrument } \\
\text { function area } \\
(\mathrm{nm})\end{array}$ & $S$ & $0.58-34.5$ & $\approx 0.4 \%$ & $\begin{array}{l}\text { Ray tracing, laser } \\
\text { scans }\end{array}$ \\
\hline
\end{tabular}

( $\left.I_{\text {detector }}\right)$ in the form (see Harder et al., 2005b):

$$
\begin{aligned}
& E\left(\lambda_{\mathrm{s}}\right)=\frac{P_{\mathrm{ESR}}\left(\lambda_{\mathrm{s}}\right)}{A_{\text {slit }} \int \alpha_{\lambda} T_{\lambda} \Phi_{\lambda} S\left(\lambda, \lambda_{\mathrm{s}}\right) \mathrm{d} \lambda} \text { or } \\
& E\left(\lambda_{\mathrm{s}}\right)=\frac{I_{\text {detector }}\left(\lambda_{\mathrm{s}}\right)}{A_{\text {slit }} \int R_{\lambda} T_{\lambda} \Phi_{\lambda} S\left(\lambda, \lambda_{\mathrm{s}}\right) \mathrm{d} \lambda} .
\end{aligned}
$$

The terms in this equation, their range, and estimated standard deviations (Taylor and Kuyatt, 1994) are listed in Table 1. Additional terms are required to account for on-orbit detector and prism degradation.

As seen from Table 1, the dominant sources of uncertainty are the optical efficiency of the ESR and to a lesser degree the uncertainty in the transmission of the prism in the 250 to $1000 \mathrm{~nm}$ range. The uncertainties for both of these components are exacerbated in the infrared. Because of the broadly changing contributions to the error budget, the best computational method to propagate errors in Equation (1) is to build a Monte-Carlo simulation that includes the error contributions determined from the component level calibrations. The findings from this simulation show that the SIM error over the 250 to $1000 \mathrm{~nm}$ range using the ESR is less than $\approx 2.8 \%$.

The terms in the SIM measurement equation were validated with a preflight end-to-end comparison using an FEL lamp with a NIST-traceable calibration that was placed $2.24 \mathrm{~m}$ from the entrance slit so the illumination of the instrument was similar to that of the Sun. This is critical for this spectrometer since the shape of the instrument function depends on the solid angle subtended by the source. The problem with this arrangement is that light intensity measured by the spectrometer is less than $2.5 \%$ of the intensity of the Sun and with a different color temperature; thus the use of the FEL lamp cannot be construed as a calibration since only the lowest few percent of the instrument's dynamic range is stimulated 
by the test. Any errors or offsets in the radiometric response of the instrument inferred from this 'calibration' would be multiplied by a factor greater than 40 when extrapolated to the operating range of the instrument for solar measurements. Nonetheless, important information about the response of the instrument can be gleaned from this activity, particularly in the wavelength range where the component level calibrations are problematic.

Figure 2 shows the outcome of illuminating the SIM instrument with an FEL lamp showing the expected output of the lamp as stated in the NIST calibration report and accounting for the $r^{-2}$ decrease in irradiance of the source for all three of the SIM A photodiode detectors. The radiant power determined by the photodiodes is the same as in Equation (1), but with:

$$
P_{\mathrm{d}}=\frac{I_{\mathrm{d}}\left(\lambda_{\mathrm{s}}\right)}{R_{\mathrm{d}}\left(\lambda_{\mathrm{s}}\right)}
$$

The power detected by each detector, $P_{\mathrm{d}}$, is the photodiode detector current $I_{\mathrm{d}}$ divided by its radiant sensitivity $\left(R_{\mathrm{d}}, \mathrm{AW}^{-1}\right)$. Note that the same calibration values used in-flight are used in the analysis of this lamp spectrum. As discussed in Harder et al. (2005b), the photodiode radiant sensitivity is determined through calibration by the ESR. In Figure 2 the spectral irradiance as determined through the SIM measurement equation reproduces the calibrated irradiance values of the lamp. Note however, that the noise contribution to the measurement is significant in some parts of the spectrum. The dashed curves in Figure 2 show the noise equivalent irradiance (NEI) of the detectors as found by measuring the noise levels of each detector and dividing by the profile integral (the denominator of Equation (1)). As an example of the noise contribution, notice that for the Vis1 photodiode (red trace of Figure 2) the signal-to-noise ratio (SNR) is less than 10 for wavelengths less than $450 \mathrm{~nm}$ where there appears to be more scatter in the data. For the photodiode signals shown in Figure 2, the ultimate noise limit is set by the performance of the sampling analog-to-digital converters (ADC) used to readout the photocurrents. These 15-bit ADC components have about 2 bits of digital noise independent of the signal level, so longer integrations of the photocurrent do not produce a square root of integration time improvement in the noise performance of the photodiodes. While this ADC-limited performance is somewhat problematic for the FEL calibration, it is adequate for solar observations; see Section 2.3 for more discussion.

The compliance of the SIM response to the FEL calibration can be determined by bivariant linear least-squares regression taking into account the measurement errors associated with individual data points (York, 1966). The FEL uncertainties used in this analysis are provided in the NIST lamp calibration data sheet, and the SIM irradiance errors are the $\pm 1 \sigma$ noise equivalent irradiance. This analysis produces a slope and intercept of $(0.988 \pm 0.004)$ and $(0.6 \pm 1.0) \times 10^{-5}$ with an $r^{2}$ coefficient of 0.9986 over the 250 to $1600 \mathrm{~nm}$ range as shown in Figure 2. Based on the uncertainties found from error propagation of the terms in Table 1 and the evidence from the FEL lamp, there was an expectation that the irradiance of SIM would be low by less than $2 \%$ but after correction would be valid to about $0.5 \%$, and the FEL comparison experiment supports this finding. The origin of the $1-2 \%$ deficit in the instrument response was addressed by performing postlaunch characterization studies with flight spare components.

\subsubsection{Post-Launch Characterization of SIM Engineering Model Components}

As seen in Table 1, the ESR optical efficiency was identified as one of the leading contributors to the uncertainty budget in the SIM calibration. Further analysis of the optical losses of the detector was performed on the SIM ESR flight spare detector. This detector 


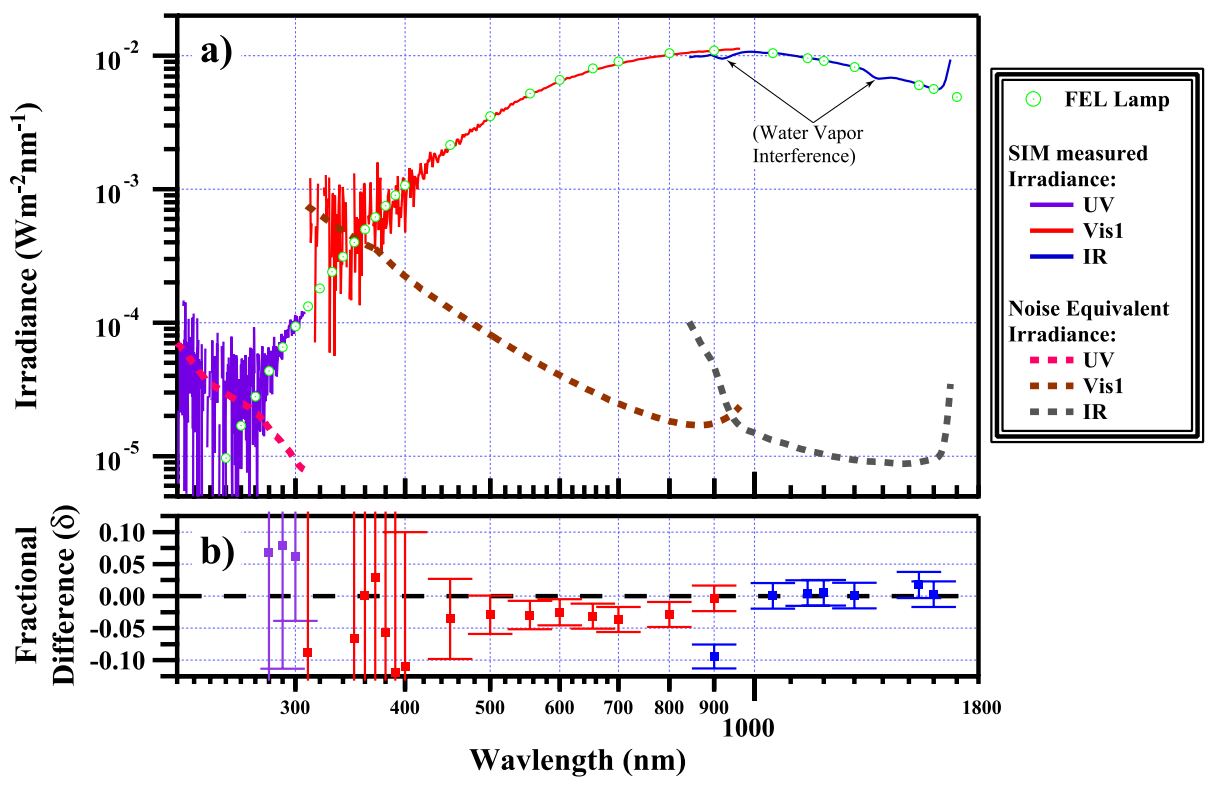

Figure 2 Comparison of SIM to a NIST certified FEL lamp as measured by the three SIM focal plane photodiodes. The lamp was located $2.24 \mathrm{~m}$ in front of the entrance slit to produce an approximate $0.5^{\circ}$ divergent light beam to illuminate the instrument in the same manner as the Sun; the lamp intensity is $\approx 2.5 \%$ of the solar intensity. The graph also shows the curves of noise equivalent irradiance to indicate where the measurements are dominated by noise. The bottom panel shows the fractional difference (SIM-FEL)/FEL over the full range of the experiment.

was manufactured and tested to the same specifications as the two flight units and represents a very close analog to the flight units; in particular, the optical properties of the nickel phosphorous black surface on the bolometer and the optical properties of the aluminized hemisphere surrounding the bolometer are representative of what is currently operating in space; see Harder et al. (2005a) for a discussion on the design of the detector. The overall efficiency of the detector in a power mode (as opposed to an irradiance mode) was investigated by stimulating the detector with NIST SIRCUS laser system and measuring the light intensity of the laser with transfer detectors traceable to the NIST cryogenic radiometer standard (Brown et al., 2004). The usage of SIRCUS as a light source has a significant advantage over a broad spectrum light, like an FEL lamp, because the calibration can occur near the top of the dynamic range of the instrument, detector illumination can be controlled to match the expected spot size, and the monochromatic radiation can be used to evaluate the spectral response and assess the contributions of scattered light. The layout of the optical system to perform this characterization is shown in Figure 3 and covers the usable responsivity range of the ESR in the 266 to $1350 \mathrm{~nm}$ wavelength region. Measurements were carried out to $2500 \mathrm{~nm}$, but the extended NIR range will not be reported here.

The SIRCUS system provides a continuously tunable light source in the UV/visible/NIR with a Ti:Sapphire laser pumped by a frequency-doubled Nd:Vanadate laser $(10 \mathrm{~W}$ at $532 \mathrm{~nm}$ ). The Ti:Sapphire laser covers the spectral range from 680 to $1050 \mathrm{~nm}$, and frequency doubling or tripling it enables tuning from 266 to $500 \mathrm{~nm}$. The CTA $\left(\mathrm{CsTiAsO}_{4}\right)$ and $\mathrm{KTP}\left(\mathrm{KTiOPO}_{4}\right)$ optical parametric oscillators tune from approximately 1050 to $1650 \mathrm{~nm}$. 


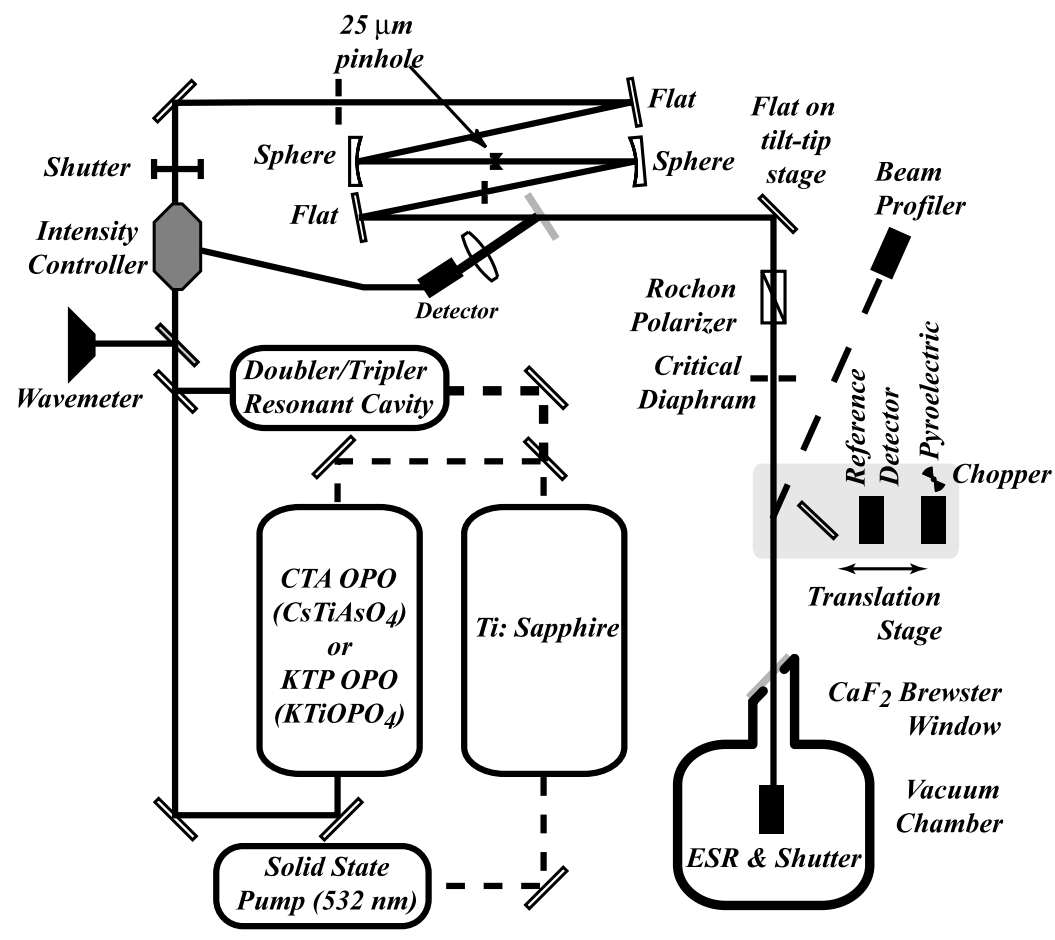

Figure 3 Experimental configuration of the NIST SIRCUS calibrated light source. This experimental arrangement allows laser wavelengths to be selected in the 266 to $1350 \mathrm{~nm}$ range.

The calibration is typically performed with about $60 \mu \mathrm{W}$ of optical power corresponding to the highest solar power observable by the SIM instrument on-orbit - well within the operating range of the lasers. The light is polarized and passes through a Brewster window with a known transmission to minimize window reflections. The ESR is operated at a pressure of $\approx 10^{-7}$ torr to ensure proper response. A flat mirror on a tilt-tip stage is used to move the light spot in two perpendicular directions to center the laser spot on the detector and ensure that the light beam is not being clipped. A beam profiler is used to monitor the spot shape to guarantee that the spot has not changed in shape and location when the laser configuration is changed. Three reference detectors were used in the experiment, depending on the target wavelength. A silicon trap detector was used for most of the visible part of the spectrum. Alternately, a $50 \mathrm{~mm}$ diameter integrating sphere was used with either silicon or InGaAs for the UV, visible, and IR out to $1.5 \mu \mathrm{m}$, a pyroelectric detector was used throughout the spectrum and at the longest wavelengths (Eppeldauer, Zeng, and Yoon, 2008). All of these detectors were calibrated against the cryogenic radiometer prior to the measurement campaign. Typically two independent reference detectors were used to determine the intensity of the laser beam. The reference detectors were outside the vacuum chamber that contained the ESR.

This optical system essentially images a $25 \mu \mathrm{m}$ pinhole onto the bolometer inside a vacuum system so that the entire radiance of the light beam under-fills the width of the bolometer and enters the reference detectors. Because the measurement is made in a power mode, no light-limiting aperture is placed in front of either the bolometer or the reference detectors. However, a critical aperture was used to prevent scattered light from reaching either 
the reference detectors or the ESR. Changing the diameter of its clear aperture and translating its location verifies that no detectable scattered light reaches the detectors. The center of the bolometer is aligned with the laser system's optical axis by using a flat mirror on a tilt-tip stage to scan the light spot across the length and width of the detector. The size of the light spot is found from both direct beam profile measurements and confirmed by analysis of the ESR cruciform scans; these two independent methods produce about the same result which is particularly important in the NIR where the spot size is measurably larger than in the visible. The laser system is stable to about $0.02 \%$ over the course of a 720 second ESR integration time and the laser intensity was measured before and after each ESR measurement. Likewise, dark signals were measured and subtracted from both the ESR and the reference detectors bracketing the laser measurement. The ESR noise spectrum was measured on multiple occasions during the campaign, and detector noise was about $0.009 \mu \mathrm{W}$ on a typical $60 \mu \mathrm{W}$ signal with a 40 second integration period giving a SNR of $\approx 7000$ for the measurement.

Figure 4a shows the results of 25 measurements spanning the 266 to $1350 \mathrm{~nm}$ range using the laboratory arrangement shown in Figure 3. The experiment was performed at the same wavelength on several occasions to determine how much data dispersion occurs when the laser configurations are changed. This accounts for most of the data scatter, since the precision and stability of both the reference detectors and the ESR have a precision at least a factor of ten smaller than what is seen in the figure. The average of the ratio of the ESR to SIRCUS is $0.987 \pm 0.005(1 \sigma)$. These individual data points fall within a band of \pm 0.005 with, at most, a weak wavelength trend. If this trend exists, it is mostly driven by two UV measurements at 266 and $300 \mathrm{~nm}$ where the non-uniformity of the detectors might contribute to the slightly reduced ratio. Figure $4 \mathrm{~b}$ demonstrates the mutual linearity of the SIRCUS system and the ESR showing a bivariant linear fit at $457.9 \mathrm{~nm}$ covering a factor of 5 of dynamic range. The radiant power from the Sun seen by the SIM detectors at this wavelength corresponds to $\approx 14 \mu \mathrm{W}$, so this calibration method allows the detector to be stimulated at a power level commensurate with the expected solar signal strength. The most important finding from this study is that the response of the SIM ESR is very weakly wavelength dependent (Figure 4a) with a very high degree of linearity with an $r^{2}$ coefficient of 0.9998 (Figure $4 \mathrm{~b}$ ), and therefore is a highly reliable space qualified reference detector for determining solar spectral variability.

Aside from the effort to use SIRCUS to analyze the radiometric response of the SIM ESR, this facility was used to study the prism spectrometer's instrument line shape (ILS). This ILS function appears directly in the measurement equation (Equation (1)) but is weighted according to other factors such as the change in the detectors responsivity across the bandpass, the transmission of the prism, and slit diffraction. For a low resolution instrument like SIM the specification of the bandpass is critical since the continuous solar structure is weighted by this profile. Light scattered by exit slit baffles and the prism polish produce light scattering that subtends a small angle relative to the central image spot (near-in scattering). This is separate in nature from instrument stray light baffling that has a much broader field of regard as viewed by the instrument's detectors. The SIM instrument has an excellent stray light rejection ratio of better than about $10^{-4}$ that was determined preflight by illuminating the spectrometer with a $1000 \mathrm{~W}$ xenon arc lamp equipped with a series of sharp-cut long pass glass filters, and then scanning the instrument through the bandpass of the filter. Any light detected at wavelengths short of the filter cut-off is stray light. The near-in scattering and ILS was analyzed preflight at selected helium - neon laser wavelengths (see Harder et al., 2005b), but a better verification was performed postflight using the more versatile SIRCUS facility especially in the NIR. 


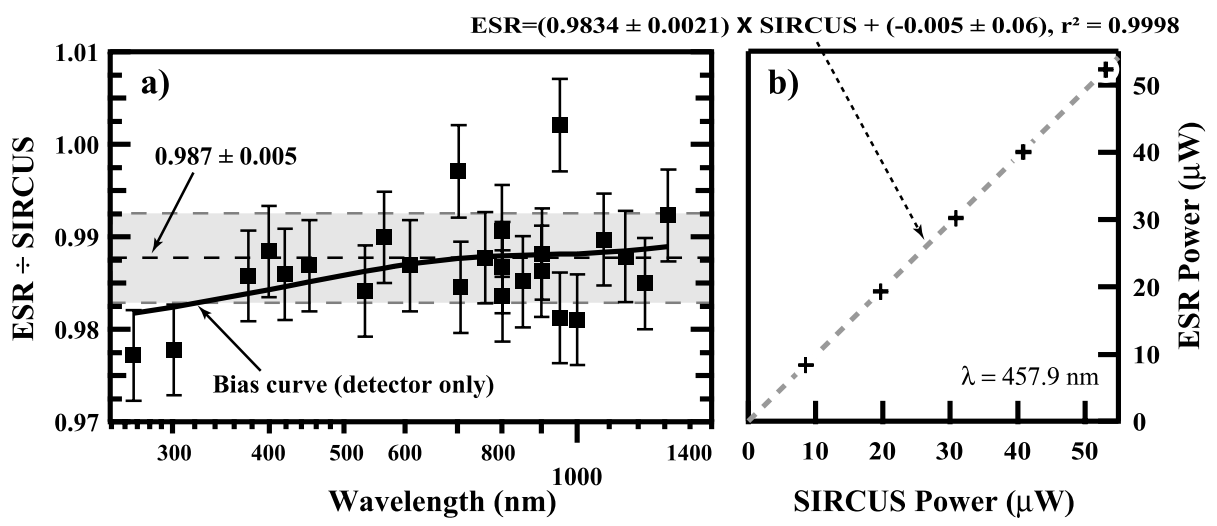

Figure 4 The SIRCUS-to-ESR comparison. Figure 4a shows the ratio of the ESR to the calibrated SIRCUS output. The data fall in a \pm 0.005 band about 0.987 . Additional uncertainty is expected in the UV where transfer standard detector uniformity becomes more problematic. Figure $4 \mathrm{~b}$ demonstrates the linearity of the ESR detector at a fixed wavelength of $457.9 \mathrm{~nm}$ and over a factor of 5 in intensity. In summary, the net result of these two calibration activities consistently indicate that the radiometric response function of SIM needs to be increased by a factor of 1.013 across the 258 to $1350 \mathrm{~nm}$ range. This value will be used in subsequent instrument comparisons, but explicitly called out when it is applied.

The basic experiment is to rotate the prism to the desired center wavelength and illuminate the detectors with a stable, monochromatic tunable laser source. The laser beam was expanded to produce a $0.5^{\circ}$ divergent beam. This test was not performed on the SIM instrument itself but on flight spares mounted on an optical breadboard with an engineering model prism drive and focal plane, with flight spare photodiodes. Individual reference wavelengths were selected to cover the entire range where the instrument detector response is non-zero across the chosen exit slit. The critical experiment that can be performed here is the verification of the reciprocity relation of scanning a tunable laser at a fixed prism rotation angle (direct scan) and scanning the prism drive with a fixed laser wavelength (indirect scan). The critical test is to verify that the ILS from the direct and indirect scans produce the same profile integral thereby demonstrating that the contributions from transmission, detector response, and diffraction are well determined for the instrument (Saunders and Shumaker, 1986).

An example of this direct/indirect comparison is shown in Figure 5 for a central laser wavelength of $646 \mathrm{~nm}$. For this experiment, a visible wavelength was selected to permit a large tuning range to perform a direct scan of the instrument profile out into the far wing of the instrument profile. A tunable ring dye laser (DCM dye) allowed for continuous tuning between 625 and $660 \mathrm{~nm}$ with an average power of $2.754 \pm 0.016 \mathrm{~mW}$. The residual plot shows very good point-to-point agreement throughout the entire passband. It is clear that there is an asymmetry in the wings of the profile (below the $10^{-2}$ intensity level); coma is observed in the red wing and scattered light is evident in the long tail of the blue wing.

Because of the scattered light contributions out of the nominally trapezoidal instrument function seen in Figure 5, additional indirect scans were performed at other wavelengths to analyze the contribution to the changes in the wings of the instrument profile. This is important because the profile integral at any wavelength is constructed from a ray trace model based on the measured geometry of the prism and locations of the exit slits relative to the entrance slit. The measured profile area can then be compared to output of the ray 


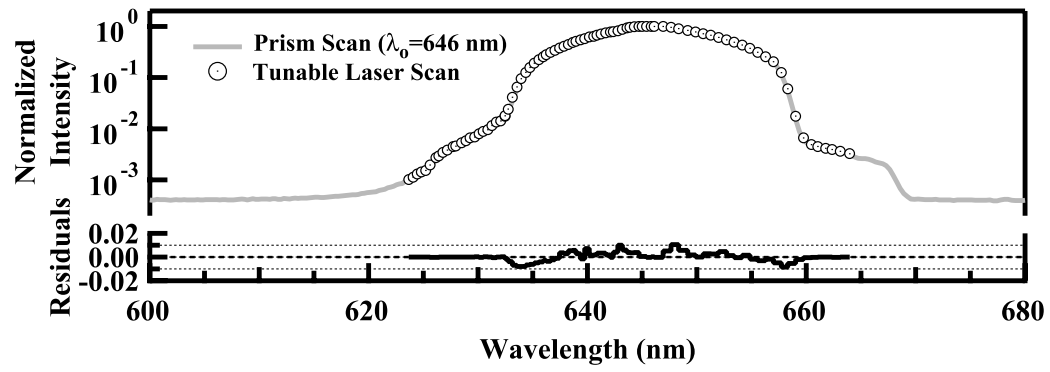

Figure 5 A comparison of the direct and indirect scans of a flight spare Féry prism. The individual data points are from the wavelengths achieved with a tunable laser for the direct scan, the gray curve is the indirect scan achieved by rotating the precision prism drive mechanism. The residual plot shows that the point-to-point difference between the two scans.

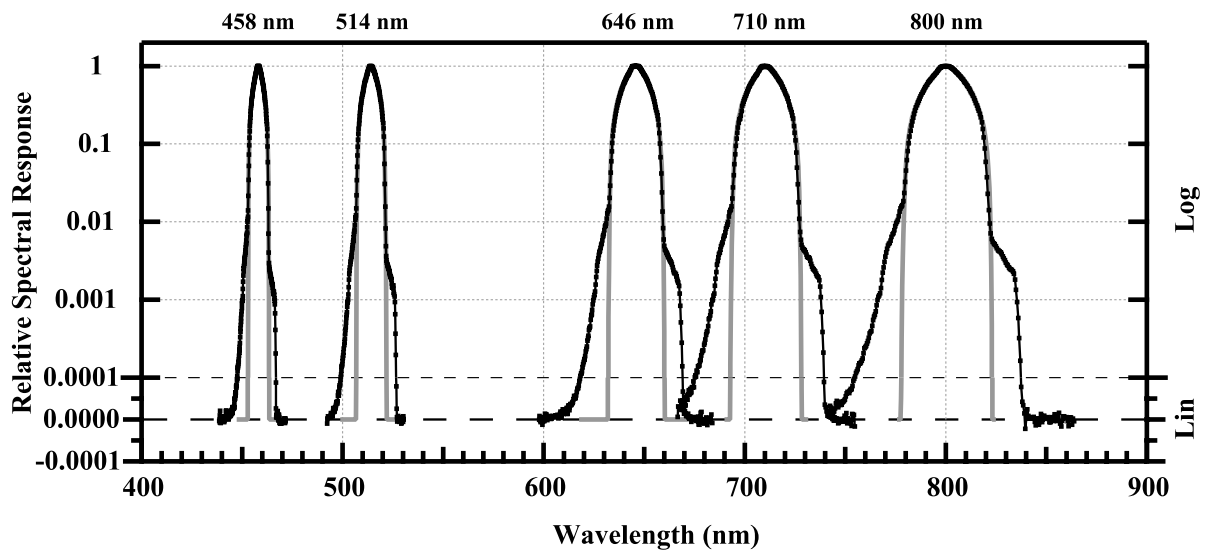

Figure 6 Measured indirect scans at five wavelengths (black symbols) and ray traced values (gray line) for the SIM instrument profile as a function of wavelength giving the relative instrument response. Graph is on a $\log$ scale from 2 to $10^{-4}$ and linear below that value. Profiles show some structure due to near-in light scattering from the exit plane baffles and optical aberrations. The areas of the measured and the ray trace modeled profiles agree to better than $5 \times 10^{-3}$.

trace model. Figure 6 shows the scans of the SIRCUS tunable laser system to measure the relative response of the system as a function of wavelength using the indirect scanning method. The plot is shown on a 4 decade log-linear scale to show the shape of the response function; it is linear below $10^{-4}$ to show the zero level of the plot. To characterize the shape, a 3.3 arc-second step was performed to generate the line profile, whereas in-flight a 25.4 arcsecond step size is normally used. In Figure 6, the gray trace corresponds to the ray traced instrument function for comparison. In all cases the area of the ray traced profile agrees with the measured profile to less than $0.5 \%$, and the agreement is improved further by generating a coma/diffraction correction factor that is applied in data processing. An important thing to note is that the profiles in Figure 6 are plotted as a function of wavelength, but when they are plotted in terms of focal plane coordinates (Harder et al., 2005b) all of these profiles overlay one another, so in this sense the instrument function shape is stationary in this coordinate system. 


\subsubsection{In-Flight Characterization}

The preflight calibration of the instrument must be maintained on-orbit with a precise prism degradation correction. The details of this procedure are described in the auxiliary material in Harder et al. (2009), but summarized here. The SIM instrument consists of four detectors (one ESR and three diodes) and two identical (mirror image) spectrometers; SIM A acquires twice-daily spectra and SIM B is the reference spectrometer that is used on a monthly basis for comparison. Sensitivity degradation predominantly arises from exposure of the prism to solar photons that are energetic enough to either directly induce compositional changes that produce an absorbing layer in the first few monolayers of the prism glass and/or transmission losses due to hard-radiation induced polymerization of trace amounts of organic material on the surface of the prism. The loss of prism transmission is essentially a surface phenomenon regardless of the detailed physical mechanism. The only assumption in the degradation analysis is that the prism transmission can be expressed as the product of an initial transmission $\left(T_{0}\right)$ times a degradation factor expressed in a logarithmic form by defining the optical thickness of the absorbing material responsible for the prism degradation. The on-orbit time and wavelength dependent prism transmission, $T(\lambda, t)$, is given by

$$
T(\lambda, t)=T_{0} e^{-\tau\left(\lambda, t-t_{0}\right)} .
$$

The time dependent attenuation of the degrading layer $\tau(\lambda, t)$ can be independently evaluated at 60 wavelengths from low-noise ESR fixed wavelength experiments performed on both SIM A and SIM B that have different levels of solar exposure in order to reconcile the solar spectral irradiance inferred by them. SIM B, the reference spectrometer, has about $24 \%$ of the solar exposure of the SIM A spectrometer and the time series of these spectrometers are compared to experimentally determine the attenuation factor in Equation (3). Over the course of the 6.5-year mission we find that the temporal dependence of $\tau$ is the same at all wavelengths (within a small uncertainty) once a time independent but wavelength dependent scaling coefficient is applied. Therefore, $\tau$ dependency on time and wavelength can be separated into a strictly wavelength dependent part, $\kappa(\lambda)$, that can be interpreted as the absorbing material(s) opacity and a strictly time dependent part, $C_{\mathrm{AorB}}(t)$, that can be interpreted as the effective thickness, or column surface density, of the absorbing material that accumulates on the surface of the SIM A or SIM B prisms over time and is different for each spectrometer. Note that $\tau$ is the only physically accessible quantity and $\kappa$ and $\mathrm{C}$ are then constructed to give $\kappa(\lambda)=\tau(\lambda, t) / C(t)$. Analysis of the time series of each of the 60 fixed wavelengths continually verifies the constancy of the values of $\kappa$ at all wavelengths and the value is refined to reflect the improved accuracy with which it can be defined from the SIM A-to-SIM B comparisons. To date we have not found any significant change in $\kappa$ within the uncertainties due to the lack of measurement simultaneity, wavelength mis-registration, and ESR noise. Using the values of $\kappa$ and $C_{\mathrm{A}}$ derived in this analysis, numerical examples of the amount of degradation can be given: after 2000 days of operation with 184 days of accumulated solar exposure the transmission has decreased by $27.5 \%, 1.27 \%$, and $0.3 \%$ at $280 \mathrm{~nm}, 656 \mathrm{~nm}$, and $1350 \mathrm{~nm}$ respectively for SIM A. In comparison, after 2000 days of operation of SIM B (39 accumulated exposure days) the transmission loss is $8.2 \%, 0.4 \%$, and $0.09 \%$ at the same wavelengths. The high dynamic range, signal-to-noise, and stability of the detectors permit very precise measurements of prism degradation.

Through the first six years of the mission, when the SIM spectrum is integrated from 200 to $1600 \mathrm{~nm}$ the SIM irradiance corrected by this method has an upward trend of about $3 \mathrm{~W} \mathrm{~m}^{-2}$ out of $1224 \mathrm{~W} \mathrm{~m}^{-2}(\approx 0.2 \%)$ thereby over-correcting the irradiance. Most likely, 
this is due to the inability to know the exact value of the relationship between the column in the A and B spectrometers. The SIM B spectrometer has $\approx 22 \%$ of the exposure of SIM A, but there is no procedure to derive an absolute correction for SIM B. To account for this, the value of the column value for SIM B $\left(C_{\mathrm{B}}\right)$ is varied so that the integrated SIM time series matches the trend in total solar irradiance (TSI) measured by the SORCE Total Irradiance Monitor. Thus, SIM is not 'normalized' to the TSI; but rather the value of one parameter (i.e. $C_{\mathrm{B}}$ ) is altered so that the long-term trends in the TSI are reproduced by SIM. This method has the advantage that the wavelength dependence of the degradation is preserved in the matching of the TSI. In this procedure, the value of $C_{\mathrm{B}}$ is decreased by $4 \%$.

\subsubsection{SIM Characterization/Calibration Summary}

The calibration of the SIM instrument is based upon performing component level calibrations of detectors, and ultimately the radiometric response of the system depends on a characterization of two quantities: the efficiency of the ESR to convert radiant power to electrical power, and the specification of the spectrometer's profile integral that determines the wavelength weighting of radiation that reaches the detectors. Comparisons with an FEL lamp employing bivariant linear least-squares regression accounting for measurement errors in both axes suggests that in the 250 to $1600 \mathrm{~nm}$ range the slope of the SIM response function is lower than the FEL lamp by a factor of $0.988 \pm 0.004$ over a factor of 300 in dynamic range with the signal about $2.5 \%$ of the expected solar signal. The flatness of the ratio over this dynamic range is suggestive of a single error in the response of the detector. This possibility was further examined at the SIRCUS facility by directly measuring the efficiency of the ESR as a function of wavelength. This was performed in the 266 to $1350 \mathrm{~nm}$ range with a light source power level commensurate with the expected solar signal levels, and at one wavelength $(457.9 \mathrm{~nm})$ the linearity of the ESR was verified compared to SIRCUS. This study indicates that the ESR measures a power level of $0.987 \pm 0.005$ relative to the suite of SIRCUS detectors. Measurements of the instrument line shape are compared to the ray trace model used in data processing, and the areas of the profile are the same to about $0.5 \%$. While both the measurements at SIRCUS and FEL study indicate that the irradiance scale of SIM is lower by $1.013 \pm 0.005 \%$, the experiment clearly indicates the value of detectorbased calibrations to probe the response of an instrument in comparison to a source-based approach such as a FEL calibration. The same conclusion concerning detector-based calibrations was drawn by Yoon, Gibson, and Barnes (2002) in their effort to improve the irradiance scale of a set of FEL lamps resulting in the reduction of the uncertainties from the previous source-based spectral irradiance scale (Walker et al., 1991) by at least a factor of 2 in the ultraviolet and visible wavelength regions and a reduction in the uncertainties in the shortwave infrared wavelength region by at least a factor of $2-10$, depending on the wavelength. Finally, a physically based degradation correction based on the comparison of two independent spectrometers with multiple overlapping detectors maintains the long-term precision to better than $0.1 \%$ over the course of the 6-year mission.

\subsection{SIM Data}

The noise equivalent irradiances for each detector are shown in Figure 2; these noise limits apply for the solar measurements as well as to the lamp calibration shown in this plot. For solar measurements, the SNR is more than adequate for determining of solar variability throughout most of the visible and NIR, where it is typically $>10000$, but in the 310 to $400 \mathrm{~nm}$ and the 200 to $240 \mathrm{~nm}$ regions the SNR drops below 1000 somewhat masking the 
solar variability signal. Therefore, time averaging or band integration is required to determine the signal associated with changes in solar irradiance in these regions. For the 200 to $1629 \mathrm{~nm}$ region, there are six spectral samples per resolution element giving adequate sampling for interpolation to intermediate wavelengths. There are two spectra per day in this wavelength regime. In the 1629 to $2400 \mathrm{~nm}$ range where the ESR is used as the primary detector for daily measurements, the sampling is reduced to 2 samples per resolution element. The SNR on the ESR makes the measurements of solar variability in this part of the spectrum marginal for determination solar variability $\left(<10^{-4}\right)$, and thus band integration and/or time averaging is required in this region as well. The twice-daily SIM photodiode data from 310 to $1620 \mathrm{~nm}$ and the daily 1620 to $2400 \mathrm{~nm}$ ESR data is available from the SORCE web site: http://lasp.colorado.edu/sorce/data/ssi_data.htm. SIM data in the 201 to $307 \mathrm{~nm}$ region are available from the authors on request.

\section{SOLSPEC Instrument and Data}

\subsection{Instrument and Calibration}

\subsubsection{Instrument}

SOLSPEC consists of three spectrometers dedicated to the measurements of the UV, visible, and NIR solar irradiance. They respectively cover the following spectral domains: 200 to $350 \mathrm{~nm}, 320$ to $850 \mathrm{~nm}$, and 800 to $2500 \mathrm{~nm}$. Each spectrometer is made of a double monochromator using holographic gratings. The six gratings are mounted on a one-piece mechanical axis that rotates by using a stepping motor. The advantage of this system is to provide a spectrometer slit function invariant as a function of grating position (see Labs et al., 1987). Furthermore, there is stable relationship between the three wavelength scales. Second order and attenuator filters are placed in the optical path as a function of the grating step number. The entrance aperture of each spectrometer is followed by a quartz diffuser to reduce the signal dependence on the Sun line of sight with respect to the instrument optical axis. Two deuterium lamps and two tungsten ribbon lamps monitor the UV, visible and IR spectrometer responsivities in-flight. The detectors consist of photomultiplier tubes for the UV and visible channels, and a PbS cell for the IR channel. In flight operations, the slit function and the relationship between the grating step number and wavelength of the observation are determined by using a helium hollow cathode lamp. Eleven minutes are required to simultaneously record the three spectra in UV, visible, and IR domains.

\subsubsection{Calibration}

Instrument calibration consists of determining the dispersion law, and establishing the dependence of the signal with the off-axis source position and its photometric response in the absolute scale. For the UV and visible spectrometers, we used 20 lines provided by laboratory hollow cathode lamps. We have verified the consistency of the two dispersion laws as both spectrometers are rotating together and follow the expected grating equations.

The absolute calibration was carried out at the Heidelberg Observatory by using its blackbody irradiance standard. It consists of a cavity made of pure graphite and heated to a calibrated temperature of $3000 \mathrm{~K}$. The blackbody temperature is known by using a pyrometer calibrated by the Physikalisch - Technische Bundesanstalt (PTB) in Berlin, Germany. The blackbody and the pyrometer are described by Mandel et al. (1998). 


\subsection{Data}

SOLSPEC was operated during the ATLAS missions onboard the Space Shuttle. For these three missions two other spectrometers also performed UV observations. Comparisons of these data gathered from the same platform and at the same time may be found in Cebula et al. (1996), and Thuillier et al. (1997, 1998a, 1998b). An extensive uncertainty analysis is given in these articles, taking into account the uncertainty of the laboratory calibration and other sources such as instrument depointing and counting rate. Accuracy is quoted to a mean of $3 \%( \pm 2 \sigma)$ with some dependence on wavelength due to the non-uniform instrument responsivity of each spectrometer. This effect is primarily due to the grating system, which generates a lower responsivity in the blue and red wing of its spectral domain, and due to the second order and attenuation filters which are intended to maintain a constant counting rate by accounting for the solar irradiance variation in the spectral domain of each spectrometer. Special care is taken during calibration in the vicinity of each filter change and in the overlap regions (i.e. UV and visible around $370 \mathrm{~nm}$ and visible and IR around $800 \mathrm{~nm}$ ) by repeating measurements and increasing the integration time. An instrument identical to SOLSPEC (named SOSP, the Solar Spectrum instrument) was flown onboard the European Retrieval Carrier (EURECA) from April 1993 to January 1994, and benefited from improved thermal conditions allowing SOSP to operate more effectively in the IR domain. The UV and visible SOLSPEC spectrometers are described in detail in Thuillier et al. (1998a) as well as their method of calibration, uncertainty analysis, and data processing.

\subsection{The ATLAS 1 and 3 Spectra}

A composite spectrum has been built (Thuillier et al., 2004) for the period of the ATLAS 1 and 3 missions. Different data sets were used to construct the spectrum:

- the Upper Atmosphere Research Satellite (UARS) measurements with the Solar Ultraviolet Spectral Irradiance Monitor (SUSIM) (Brueckner et al., 1993) and the SOLar STEllar Irradiance Comparison Experiment (SOLSTICE) (Rottman, Woods, and Sparn, 1993)

- the three ATLAS missions with a second SUSIM, similar to the UARS instrument (Floyd et al., 2002), the Shuttle Solar Backscatter Ultraviolet (SSBUV) (Cebula et al., 1996) and SOLSPEC

- the EURECA mission with SOSP

The composite spectra were made using the above measurements organized as below:

- between 200 and $400 \mathrm{~nm}$, SSBUV, SUSIM and SOLSPEC data from the ATLAS mission, and SOLSTICE and SUSIM from UARS

- between 400 and $800 \mathrm{~nm}$ : SOLSPEC data

- above $800 \mathrm{~nm}$, SOSP IR data

This choice was based on the best available data. The two composite spectra were extended below $200 \mathrm{~nm}$, but not used in the present study.

For comparing SIM with SOLSPEC data, we need a spectrum covering the 200 to $1350 \mathrm{~nm}$ range. The merging of the UV and visible data has been made with the ATLAS 3 observations that, furthermore, cover a period of solar activity closer to the SIM conditions of observations. The instrument resolution is shown if Figure 1 relative to the SIM instrument. The ATLAS 3 spectrum has a quoted resolution of $0.25 \mathrm{~nm}$ from $200-400 \mathrm{~nm}$ with a spectral sampling of $0.05 \mathrm{~nm}$, and in the visible the quoted resolution is $0.5 \mathrm{~nm}$ with a variable sampling of $0.2-0.6 \mathrm{~nm}$. The resolution and sampling of the ATLAS 3 composite spectrum is adequate for slit function convolution over the full wavelength range used in the next section. 


\section{Instrument Comparisons in the UV-Visible Wavelength Range}

In this section, we will compare the visible and NIR for SIM and ATLAS 3, and in the ultraviolet for SIM, ATLAS 3, and UARS and SORCE SOLSTICE (SOLSTICE I and II, respectively). In all cases the ATLAS 3 spectrum will be used as the reference. The SOLSTICE I measurements are co-temporal with the ATLAS 3 measurements on 27 November 1996 and the SORCE SIM and SOLSTICE II measurements are on the same day (29 November 2004), thus covering similar conditions in two separate solar cycles. The day selected in the SORCE epoch was chosen because of the similarity of solar activity and because it was during a time of particularly high quality SIM ESR data. Acquiring the full ESR spectrum requires 15 consecutive orbits, and spacecraft operations were extremely stable for this particular day with minimal disruptions from South Atlantic Anomaly effects. For SORCE era spectra on 29 November 2004, the F10.7 radio flux and sunspot number were 108.4, 28; and for the UARS/ATLAS 3 time period on 27 November 1996 the values were 100.0 and 45.0 respectively.

The comparison is achieved by performing a realistic slit function convolution of the higher resolution ATLAS 3, SOLSTICE I and II spectra with the lower and variable SIM instrument function. The SIM instrument function can be computed at every wavelength step of the higher resolution instrument by ray trace analysis (see Section 2.2.2). In practice, the ray trace analysis was done at a series of wavelengths spaced on a constant index of refraction grid of the prism glass, which is proportional to a uniform prism rotation angle grid. The response of the instrument function is very smooth with wavelength, so a grid of about 100 instrument functions can be ray traced and the trapezoidal approximation of the instrument function can be determined at any wavelength by interpolation. For each point in the higher resolution spectrum an instrument function is calculated, and the convolution is performed in the neighborhood of that wavelength. The effectiveness of the convolution can be checked by numerically integrating the convolved and un-convolved spectra over wavelength and demonstrating that the integrated area has not been changed by the convolution procedure. This convolved spectrum can then be re-interpolated onto the native wavelength grid of the SIM instrument and compared wavelength-by-wavelength. Comparisons are achieved by analysis of the fractional differences $(\delta)$ of irradiance between the target instruments (SIM, SOLSTICE I and II) and ATLAS 3 spectra $\left(E_{\text {Target }}\right.$ and $\left.E_{\mathrm{ATL}}\right)$ at each wavelength step $(\lambda)$ defined by:

$$
\delta(\lambda)=\frac{\left(E_{\text {Target }}(\lambda)-E_{\mathrm{ATL}}(\lambda)\right)}{E_{\mathrm{ATL}}(\lambda)}
$$

\subsection{Comparison of SIM and ATLAS 3 in the 258 to $1350 \mathrm{~nm}$ Range}

This comparison is done over the ESR operational range of 258 to 1350 using the ESR in a phase sensitive detection mode with the shutter operating at $0.05 \mathrm{~Hz}$ and 2 shutter cycles per wavelength step (see Harder et al. (2005b) for details on the ESR operation). For these spectral scans, three samples per resolution element are used. For this comparison, the SIM irradiance derived from pre-fight calibration parameters is used without applying the corrections discussed in Section 2.2; the application of this correction is discussed in Section 4.3. In Figure 7a, the integrated areas of the two spectra from 258.125 to 1356.92 agree to $-1.40 \%\left(-16.1 \mathrm{~W} \mathrm{~m}^{-2}\right.$ out of $\left.1143.75 \mathrm{~W} \mathrm{~m}^{-2}\right)$ showing that there is significant agreement in the overall calibration. Figure $7 \mathrm{~b}$ shows that the spectral feature registration is excellent between the convolved ATLAS 3 spectrum and SIM with no indication of a 


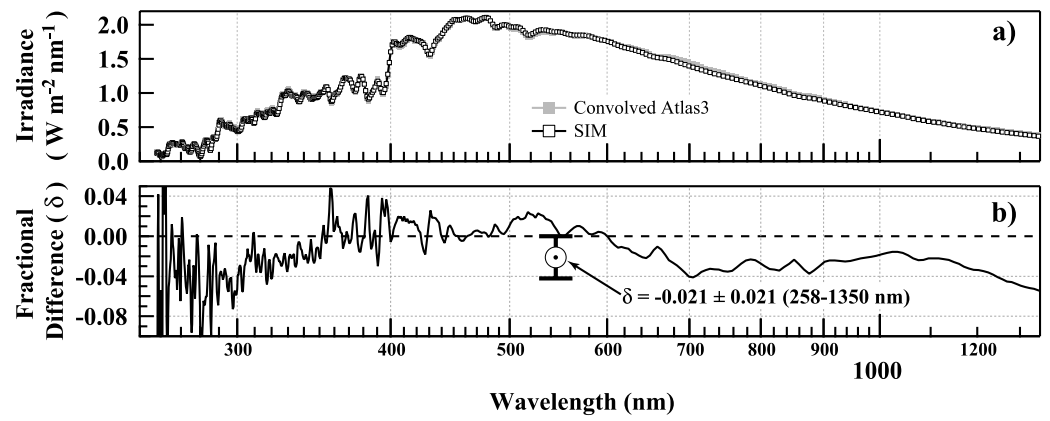

Figure 7 Comparison of SIM with the SIM instrument function convolved ATLAS 3 composite. The graphs span the 258 to $1350 \mathrm{~nm}$ range. Panel (a) demonstrates the effectiveness of the convolution to convert the ATLAS 3 spectrum to an equivalent SIM spectrum. Panel (b) shows the fractional difference as defined in Equation (4). The structure seen at the shorter wavelengths appears mostly at extrema of the solar features rather than at the locations where the spectrum is changing most rapidly; thus shifts in the reported wavelength scales between the spectra are not the dominant cause of the fluctuations observed in the fractional difference. The plot symbol in the center of panel (b) is the average fractional difference with its $k=1$ error bar.

wavelength shift. The point-to-point structure seen in Figure $7 \mathrm{~b}$ is due to a number of contributions including noise in both measurements, possible inaccuracies in the convolution process in locations where there is densely spaced solar Fraunhofer structure, and possible scattered light contamination since the largest excursions occur in the tops and bottoms of the solar features. The lower frequency variations are mostly due to differences in the radiometric calibration. Over the full 258 to $1350 \mathrm{~nm}$ range the average fractional difference of SIM from the ATLAS 3 measurement is $-0.021 \pm 0.021$; thus on average, SIM is $2.1 \%$ lower than ATLAS 3 with a $\pm 2.1 \%(k=1)$ uncertainty.

\subsection{Comparison of SIM, SOLSTICE I and SOLSTICE II with ATLAS 3 in the 220 to $310 \mathrm{~nm}$ Region}

For the SORCE mission, the data from the higher resolution SOLSTICE II spectrometer are used for daily measurements of spectral variability in the MUV and the generation of the Mg II index used for space weather applications (Snow et al., 2005). The calibration of the SOLSTICE II was performed at the NIST SURF III facility (Synchrotron Ultraviolet Radiation Facility; McClintock, Snow, and Woods, 2005; Arp et al., 2000) and has the most comprehensive preflight calibration of the four instruments in this part of the spectrum. The overall absolute accuracy of its calibration is valid to about $2 \%$ in the 200 to $300 \mathrm{~nm}$ range and has a resolution of $0.1 \mathrm{~nm}$ with 3 points per resolution element sampling. The UARS SOLSTICE I F-channel instrument (Rottman, Woods, and Sparn, 1993; Woods, Rottman, and Ucker, 1993; Woods et al., 1996) has a SURF II based calibration that is estimated to be accurate to $3 \%(k=1)$. The instrument covers the wavelength range 170 to $315 \mathrm{~nm}$ with a resolution of $0.2 \mathrm{~nm}$ and a sampling of 3 points per resolution element. The SIM calibration for the UV photodiodes is based on the prism transmission calibration and the ESR responsivity calibration down to a wavelength of $\approx 258 \mathrm{~nm}$. For the shorter wavelengths the calibration is tied to the SORCE SOLSTICE measurement. The higher resolution daily UV measurements of SOLSTICE II are considered a more useful data product, so extensive absolute calibration of the SIM UV were not pursued in the 200 to $260 \mathrm{~nm}$ region. Nonetheless, this part of the UV spectrum from SIM is considered important since the continuity of degradation corrections must be seen to smoothly change as a function of 


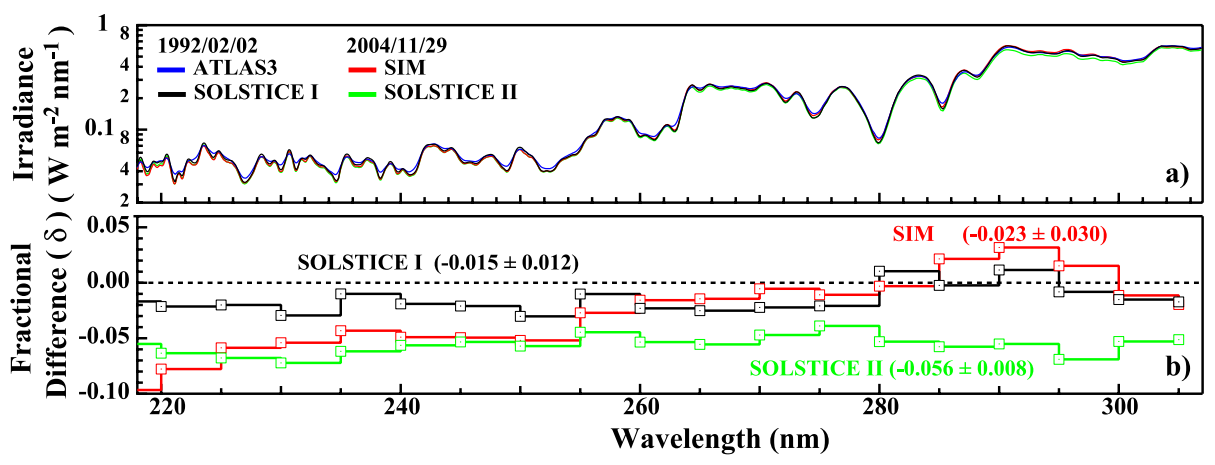

Figure 8 Comparison of SIM, SOLSTICE I, and SOLSTICE II relative to ATLAS 3. Panel (a) shows the response of the 4 instruments at the resolution of SIM. Panel b shows the fractional differences as a function of wavelength integrated into $5 \mathrm{~nm}$ bins and the fractional difference statistics are shown in the plot labels. The integrated irradiances over the 220 to $307 \mathrm{~nm}$ range for the four spectra in units of $\mathrm{W} \mathrm{m}^{-2}$ are: 18.48 (SIM), 17.50 (SOLSTICE II), 18.53 (ATLAS 3), 18.35 (SOLSTICE I).

wavelength. Time series comparisons between the two independent SIM and SOLSTICE spectrometers can be used to validate the solar cycle trends, and this portion of the solar spectrum has a significant contribution to the variability in TSI (Harder et al., 2009) and must be included in comparisons with the SORCE Total Irradiance Monitor (TIM). Because the SNR of the SIM UV channel drops to about 50 at $220 \mathrm{~nm}$, the noise level at the shortest wavelengths affects the comparison, so the comparison will be limited to wavelengths greater than this value.

Figure 8a shows a comparison of the SIM, SOLSTICE I, and SOLSTICE II measurements relative to the ATLAS 3 spectrum. As in the case of the visible/IR comparison in Figure 7, the higher resolution spectra are convolved with the SIM instrument function and over-plotted. Since the fractional difference is sensitive to wavelength misalignment, the SIM wavelength scale was adjusted to match the wavelength scale of SOLSTICE with a quadratic function determined from a least-squares fit of the first derivative of the two spectra; in this way, the locations of the points of greatest slope are smoothly co-aligned over the full wavelength range. This procedure produces a maximum wavelength shift of $0.152 \mathrm{~nm}$ at $307 \mathrm{~nm}$ prior to computing the fractional difference. This adjustment is within the capability of determining the absolute scale of the SIM prism drive as stated in Table 1. Figure $8 \mathrm{~b}$ shows the fractional differences of SIM, SOLSTICE I, and SOLSTICE II relative to ATLAS 3. To compare the spectra, the data are integrated over $5 \mathrm{~nm}$ bins prior to computing the fractional difference. This was done because the ATLAS 3 data show less feature contrast relative to the other three instruments. This low feature contrast would result in large fluctuations in the fractional difference if performed on a point-by-point basis, as was done in Figure 7. The data in Figure 8 are shown at the SIM resolution, but even at the reported resolution of the ATLAS 3 data, no single Gaussian filter smoothing parameter can be applied to the higher resolution SOLSTICE II data to bring the two spectra into a common level of feature contrast over the entire spectral range. The block integration shown in Figure 8 emphasizes differences in the absolute calibration rather than spectral feature mis-registration.

For SOLSTICE I, the fractional difference in the 220 to $307 \mathrm{~nm}$ range is $-5.6 \%$ with $\pm 0.8 \%$ ( $k=1)$ deviation about this mean. As described by Thuillier et al. (2004), in this wavelength region, the ATLAS composite is based on the average of five datasets (SOLSTICE I, UARS SUSIM, SSBUV, ATLAS SUSIM, and SOLSPEC). Woods et al. (1996) 
compared these datasets for 15 April 1993 and found that the two UARS instruments agreed to about $5 \%$. The average uncertainty of the composite rose to about $10 \%$ when ATLAS SUSIM and SSBUV were included. Therefore, the level of agreement shown in Figure 8 is entirely within the expected uncertainty of the ATLAS 3 composite.

For SIM, its independent calibration in the 258 to $307 \mathrm{~nm}$ range agrees with ATLAS 3 to $+5.4 \pm 2.4 \%(k=1)$, but in the full 220 to $307 \mathrm{~nm}$ range the agreement is $-2.3 \pm 3 \%$, and below $230 \mathrm{~nm}$ the agreement rolls off due to the limited SNR of the measurement at the shortest wavelengths. The SOLSTICE I comparison shows good agreement $(-1.5 \pm 1.2 \%)$ and appears to have a constant ratio with respect to ATLAS 3 throughout the full MUV range suggesting that they differ by a constant radiometric factor. In this analysis, the irradiance calibrations for all four instruments are valid to about the 5\% level with the SOLSTICE I and II instruments showing better agreement with less than $\pm 1.2 \%$ standard deviation.

\subsection{Final Corrections of the SIM Instrument out to $2400 \mathrm{~nm}$}

Sections 4.2 and 4.3 showed that SIM and the ATLAS 3 spectra agree to $-2.1 \pm 2 \%$ in the visible and NIR $(258-1350 \mathrm{~nm})$ using the ESR as the detector and 5\% in the overlapping ultraviolet $(220-300 \mathrm{~nm})$. Integrated over the full 220 to $1350 \mathrm{~nm}$ range, the spectrometers account for $84 \%$ of the TSI $\left(1143.8-1360.75 \mathrm{~W} \mathrm{~m}^{-2}\right)$. Application of the correction factor determined from the FEL and SIRCUS calibration activities improves the level of agreement between SIM and SOLPEC, but these corrections are applicable only in the 258 to $1350 \mathrm{~nm}$ range. The portion of the spectrum from 1350 to $2400 \mathrm{~nm}$ has a significant contribution to the TSI and contains the critically important $1623 \mathrm{~nm} \mathrm{H}^{-}$opacity minimum region of the spectrum (Fontenla et al., 2006). Figure 9a shows the fractional difference comparison between SIM and ATLAS 3 including this longer wavelength region and indicates that SIM continues to be systematically lower reaching a value of $-8 \%$ near $1500 \mathrm{~nm}$ and remains at that value out to $2400 \mathrm{~nm}$. This systematic difference between uncorrected SIM and ATLAS 3 generates a deficit in the integrated spectral irradiance of $30.5 \mathrm{~W} \mathrm{~m}^{-2}$ in the 258 to $2400 \mathrm{~nm}$ range that cannot be accounted for by other means. The SRPM (Solar Radiation

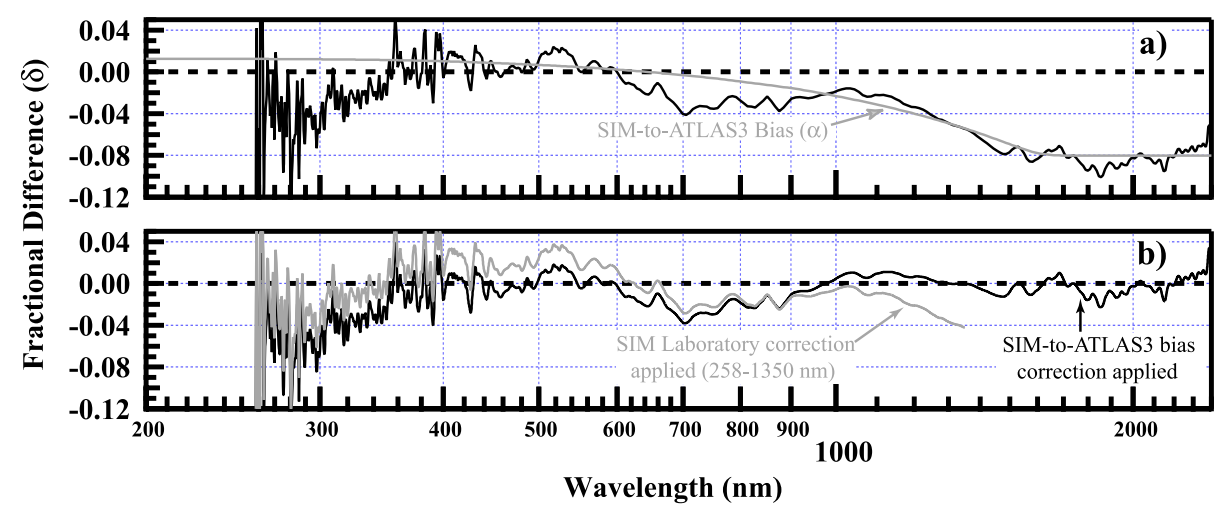

Figure 9 Panel (a) is the fractional difference over the full 258 to $2400 \mathrm{~nm}$ range for SIM and ATLAS 3 prior to application of the bias correction. This panel shows the systematic trend of SIM relative to SOLSPEC for the longer wavelengths. The SIM-to-SOLSPEC bias curve $(\alpha)$ is shown in gray. Panel (b) show the fractional difference between SIM and SOLSPEC after the SIM spectrum is divided by 0.987 , the factor found in Section 2.2 (gray trace) and after it is divided by $(1+\alpha)$, the SIM-to-SOLSPEC correction factor (black trace). This latter correction factor is used for the published SIM irradiance values at this particular time. 
Table 2 Comparison of SIM and SOLSPEC with the application of irradiance corrections. The table shows fractional differences $(\delta) \pm 1 \sigma$ standard deviations and the band integrals (Int.) over the stated wavelength range in units of $\mathrm{W} \mathrm{m}^{-2}$.

\begin{tabular}{|c|c|c|c|c|c|c|}
\hline & \multicolumn{2}{|l|}{$258-1350 \mathrm{~nm}$} & \multicolumn{2}{|l|}{$1350-2400 \mathrm{~nm}$} & \multicolumn{2}{|l|}{$258-2400 \mathrm{~nm}$} \\
\hline & $\bar{\delta}$ & Int. & $\bar{\delta}$ & Int. & $\bar{\delta}$ & Int. \\
\hline $\begin{array}{l}\text { Component level } \\
\text { calibration }\end{array}$ & $-0.021 \pm 0.021$ & 1125.17 & $-0.080 \pm 0.010$ & 172.23 & $-0.050 \pm 0.033$ & 1297.40 \\
\hline $\begin{array}{l}\text { Section } 2.2 \\
\text { correction }\end{array}$ & $-0.008 \pm 0.021$ & 1139.99 & - & - & - & - \\
\hline $\begin{array}{l}\text { SIM-to-ATLAS } \\
\text { bias correction }\end{array}$ & $-0.008 \pm 0.019$ & 1131.90 & $-0.003 \pm 0.009$ & 185.98 & $-0.005 \pm 0.015$ & 1317.88 \\
\hline SOLSPEC & - & 1141.09 & - & 186.81 & - & 1327.90 \\
\hline
\end{tabular}

Physical Model; Fontenla et al., 2006) model estimates that the long wavelength portion of the TSI greater than $2400 \mathrm{~nm}$ is $49.8 \mathrm{~W} \mathrm{~m}^{-2}$ making the deficit in the SIM calibration too large to be commensurate with a canonical solar minimum value of $1360.5 \mathrm{~W} \mathrm{~m}^{-2}$. The gray curve in Figure 9a shows the lowest order curve (referred to as $\alpha$ in the figure) that is consistent with both the independent SIM calibration in the 258 to $1350 \mathrm{~nm}$ range and also accounts for the infrared difference between SIM and ATLAS 3. Figure 9b shows the fractional difference between SIM and ATLAS after the application of the Section 2.2 corrections (gray trace) and the fractional difference after division of the SIM spectrum by a factor of $(1+\alpha)$. We have therefore adopted this bias correction factor for SIM based on the ATLAS 3 comparison and use it for the published SIM data. The analysis of these correction factors is summarized in Table 2 for changes in fractional difference and integrated power in specific bands. It must be emphasized that without any modification of the preflight calibration the two instruments agree to $-2.1 \pm 2.1 \%$. Application of the corrections described in Section 2.2 account for the $-2.1 \%$ bias in the preflight calibration, and the application of the SOLSPEC-derived $(1+\alpha)$ factor fixes a systematic error in the SIM calibration in the $1350-2400 \mathrm{~nm}$ range but does not alter the level of agreement found in the $200-1350 \mathrm{~nm}$ range.

\section{Summary and Conclusions}

In this paper we have discussed in detail the calibration and comparative analysis of two instruments; the SORCE SIM and the ATLAS 3. While the ATLAS 3 instrument is actually a composite, the majority of the visible and NIR portions of the spectrum are from the SOLSPEC and SOSP spectrometers that have a common design heritage. The end-to-end calibration of SOLSPEC is based on the $3000 \mathrm{~K}$ blackbody source at the Heidelberg Observatory with the blackbody temperature determined from a pyrometer calibrated at PhysikalischTechnische Bundesanstalt (PTB). The radiometric response of the SIM instrument is done by performing unit level calibrations of instrument components and constructing a measurement equation. The overall response of the system can then be verified through system level tests with an FEL lamp, and in the case of the ESR postflight analysis through the NIST SIRCUS facility. In these tests, it was determined that the SIM instrument has a response $1.3 \%$ lower than the findings from the preflight characterization. Tests at SIRCUS confirm that this lower response is due to a lower but spectrally flat detector efficiency. 
Comparisons are made by first performing detailed slit function convolution of the higher resolution instruments to the SIM resolution. After this convolution the wavelength registration of the two instruments can be checked and (if needed) corrected so fractional difference between the two spectra are representative of differences in radiometric response and instrument performance. In the 258 to $1350 \mathrm{~nm}$ range, the agreement between SIM and SOLSPEC is excellent: the two instruments agree to $\pm 2.1 \%(k=1)$ and with division by the 0.987 correction factor found in Section 2.2, the bias between the two instruments is less than $1 \%$. In the ultraviolet ( $220 \mathrm{~nm}$ to $307 \mathrm{~nm}$ ) the agreement is about $5 \%$, but fractional differences reveal other instrument-related differences that must be noted. Analysis in this part of the spectrum was aided by including the UARS and SORCE SOLSTICE spectra to the comparison. Compared to the spectra produced by other three instruments the ATLAS 3 composite shows less feature contrast and the resulting local excursions tend to mask the radiometric differences in spectral ratios. The SIM calibration is tied to the SOLSTICE calibration for wavelengths less than $258 \mathrm{~nm}$, but for the longer wavelengths that are based on the ESR calibration, the SIM radiometric response tends to be $1-2 \%$ higher than SOLSTICE I and ATLAS 3 and $7.5 \%$ higher than SORCE SOLSTICE. Below about $225 \mathrm{~nm}$ the compromised signal-to-noise ratio of the SIM UV channel affects the quality of the comparison.

The most important difference between the SIM and ATLAS 3 spectra occurs in the NIR. Figure 9a shows that the SIM instrument tends to be systematically lower than the SOLSPEC instrument; the disagreement is about $8 \%$ for wavelengths greater than $1500 \mathrm{~nm}$. As discuss in Section 4.3, this decreased level of agreement amounts to $30.5 \mathrm{~W} \mathrm{~m}^{-2}$, a difference too large to be accounted for by any other means besides an instrumental effect. It is for this reason that a bias correction based on the SIM-to-SOLSPEC comparison is applied to the SIM data. Analysis of the ISS SOLAR instrument (Thuillier et al., 2009) and the pending calibration of the next generation of the SIM instrument for the NPOESS TSIS (National Polar Orbiting Environment Satellite System, Total and Spectral Solar Irradiance Sensor) mission will explicitly address this calibration discrepancy and provide the basis for the calibration of the next generation SIM instrument.

Acknowledgements This research was supported by the NASA Solar Radiation and Climate Experiment, contract NAS5-97045. The authors would like to acknowledge Nathaniel Miller (formerly at LASP) and Alan Smith (NIST) for their support at the NIST SIRCUS facility, and Vanessa George for manuscript preparation.

SOLSPEC investigation is supported by the Centre National d'Etudes Spatiales (France), the Centre National de la Recherche Scientifique (France), the Federal Office for Scientific, Technical and Cultural Affairs (Belgium), and the Bundesministerium für Forschung und Technologie (Germany). The developing institutes are LATMOS (formerly Service d'Aéronomie du CNRS, the Institut d'Aéronomie Spatiale de Belgique and the Landessternwarte (Germany). The SOLSPEC absolute calibration has been carried out with the blackbody of the Landessternwarte (Germany).

Open Access This article is distributed under the terms of the Creative Commons Attribution Noncommercial License which permits any noncommercial use, distribution, and reproduction in any medium, provided the original author(s) and source are credited.

\section{References}

Arp, U., Friedman, R., Furst, M.L., Makar, S., Shaw, P.-S.: 2000, Metrologia 37, 357.

Brown, S.W., Eppeldauer, G., Rice, J.P., Zhang, J., Lykke, K.: 2004, In: Barnes, W.L., Butler, J.J. (eds) Earth Observing Systems IX, Proc. SPIE 5542, 363.

Brueckner, G.E., Eldow, K.L., Floyd, L.E., Lean, J.L., VanHoosier, M.E.J.: 1993, J. Geophys. Res. $98,10695$.

Chamberlin, P.C., Woods, T.N., Crotser, D.A., Eparvier, F.G., Hock, R.A., Woodraska, D.L.: 2009, Geophys. Res. Lett. 36, L05102. 
Cebula, R.P., Thuillier, G., VanHoosier, M.E.J., Hilsenrath, E., Hersé, M., Simon, P.C.: 1996, Geophys. Res. Lett. 23, 2289.

Eppeldauer, G.P., Zeng, J., Yoon, H.W.: 2008, In: Andresen, B.F., Fulop, G.F., Norton, P.R. (eds) Infrared Technology and Applications XXXIV, Proc. SPIE 6940, 694036.

Floyd, L.E., Prinz, D.K., Crane, P.C., Herring, L.C.: 2002, Adv. Space Res. 29, 1957.

Fontenla, J.M., Avrett, E., Thuillier, G., Harder, J.: 2006, Astrophys. J. 639, 441.

Harder, J.W., Lawrence, G., Fontenla, J., Rottman, G., Woods, T.: 2005a, Solar Phys. 203, 141.

Harder, J.W., Fontenla, J., Lawrence, G., Woods, T., Rottman, G.: 2005b, Solar Phys. 203, 169.

Harder, J.W., Fontenla, J.M., Pilewskie, P., Richard, E.C., Woods, T.N.: 2009, Geophys. Res. Lett. 36, L07801.

Labs, D., Neckel, H., Simon, P., Thuillier, G.: 1987, Solar Phys. 107, 203.

Lean, J.L., Rottman, R.J., Kyle, H.L., Woods, T.N., Hickey, J.R., Puga, L.C.: 1997, J. Geophys. Res. 102, 29939.

Mandel, H., Labs, D., Thuillier, G., Hersé, M., Simon, P.C., Gillotay, D.: 1998, Metrologia 35, 697.

McClintock, W.E., Snow, M., Woods, T.N.: 2005, Solar Phys. 230, 259.

Pagaran, J., Weber, M., Burrows, J.P.: 2009, Astrophys. J. 700, 1884.

Rind, D., Lean, J., Lerner, J., Lonergan, P., Leboissitier, A.: 2008, J. Geophys. Res. 113, D24103.

Rottman, G.J., Woods, T., Sparn, T.: 1993, J. Geophys. Res. 98, 10667.

Saunders, R.D., Shumaker, J.B.: 1986, Appl. Opt. 25, 20.

Snow, M., McClintock, W.E., Woods, T.N., White, O.R., Harder, J.W., Rottman, G.: 2005, Solar Phys. 230 , 325.

Taylor, B.N., Kuyatt, C.E.: 1994, Guidelines for Evaluating and Expressing the Uncertainty of NIST Measurement Results. NIST Technical Note 1297, 1994 Edition.

Thuillier, G., Hersé, M., Simon, P.C., Labs, D., Mandel, H., Gillotay, D.: 1997, Solar Phys. 171, 283.

Thuillier, G., Hersé, M., Simon, P.C., Labs, D., Mandel, H., Gillotay, D.: 1998a, Solar Phys 177, 41.

Thuillier, G., Hersé, M., Simon, P.C., Labs, D., Mandel, H., Gillotay, D.: 1998b, Metrologia 35, 697.

Thuillier, G., Hersé, M., Labs, D., Foujols, T., Peetermans, W.: Gillotay, et al.: 2003, Solar Phys. $214,1$.

Thuillier, G., Woods, T.N., Cebula, R., Hilsenrath, E., Hersé, M., Labs, D.: 2004, In: Pap, J., Fox, P. (eds) Solar Variability and its Effect on Climate, AGU Geophys. Monograph 141, 171.

Thuillier, G., Foujols, T., Bolsée, D., Gillotay, D., Hersé, M., Peetermans, W., et al.: 2009, Solar Phys. 257, 185.

Walker, J.H., Saunders, R.D., Jackson, J.K., Mielenz, K.D.: 1991, J. Res. Natl. Inst. Stand. Technol. $96,647$.

Warren, D.A., Hackwell, J., Gutierrez, D.: 1997, Opt. Eng. 36, 1174.

Woods, T.N., Rottman, G.J., Ucker, G.J.: 1993, J. Geophys. Res. 98, 10679.

Woods, T.N., Prinz, D., Rottman, G., London, J., Crane, P., Cebula, R., et al.: 1996, J. Geophys. Res. 101, 9541.

Woods, T.N., Chamberlin, P.C., Harder, J.W., Hock, R.A., Snow, M., Eparvier, F.G., Fontenla, J., McClintock, W.E., Richard, E.C.: 2009, Geophys. Res. Lett. 36, L01101.

Yoon, H.W., Gibson, C.E., Barnes, P.Y.: 2002, Appl. Opt. 41, 5879.

York, D.: 1966, Can. J. Phys. 44, 1079. 